\title{
Metachromatic leukodystrophy: Characterization of two (p.Leu433Val, p.Gly449Arg) arylsulfatase A mutations
}

\author{
YANGYANG WANG, XIANG CHEN, CHAN LIU, SHAMIN WU, \\ QINGFENG XIE, QUAN HU, SHAN CHEN and YIWEI LIU

\begin{abstract}
Department of Rehabilitation, The Second Affiliated Hospital and Yuying Children's Hospital of Wenzhou Medical University, Wenzhou, Zhejiang 325000, P.R. China
\end{abstract}

Received October 12, 2018; Accepted April 18, 2019

DOI: $10.3892 /$ etm. 2019.7760

\begin{abstract}
Metachromatic leukodystrophy disorder (MLD) is an autosomal recessive lysosomal storage disease. The disease is primarily caused by a deficiency in the enzyme arylsulfatase A (ASA), which is encoded by the ARSA gene. A total of 254 mutations have been reported in different populations. The present study aimed to detect causative gene mutations in an atypical case presenting with attention deficit hyperactivity disorder through whole-exome sequencing. Of note, the patient's mother is from a consanguineous family. Compound heterozygous variants $($ c.1297C $>\mathrm{G})+(\mathrm{c} .1345 \mathrm{G}>\mathrm{A})$ $[($ p.Leu433Val $)+$ (p.Gly449Arg $)]$ were identified in exon 8 in the ARSA gene of the pediatric patient. The two missense mutations identified have not been previously reported, to the best of our knowledge. Furthermore, an in silico analysis and multiple phylogenetic tree analyses of ARSA homologs were performed to predict the effects of the two novel mutations. Serial changes were observed in the patient with MLD at follow-up visits over 6 years. However, brain MRI images demonstrated no notable progression and the number of ASA enzymes was stable. Also, the results of neurodevelopmental assessment showed that the patient was diagnose with ADHD. These data may offer a potential explanation of the
\end{abstract}

Correspondence to: Professor Xiang Chen, Department of Rehabilitation, The Second Affiliated Hospital and Yuying Children's Hospital of Wenzhou Medical University, 109 Xueyuanxi Road, Wenzhou, Zhejiang 325000, P.R. China

E-mail: chenxiangnj@aliyun.com

Abbreviations: MLD, metachromatic leukodystrophy; ASA, arylsulfatase A; WES, whole-exome sequencing; ADHD, attention deficit hyperactivity disorder; MRI, magnetic resonance imaging; DQ, development quotient; IQ, intelligence quotient; EMG, electromyography; WAIS-III, Wechsler intelligence scale-third edition; SNV, single-nucleotide variant; T1WI, T1-weighted imaging; $\mathrm{CNV}$, copy number variation

Key words: metachromatic leukodystrophy, arylsulfatase A, mutation analysis, whole-exome sequencing, attention deficit hyperactivity disorder genotype-phenotype correlation in MLD and enhance the spectrum of mutations associated with the condition.

\section{Introduction}

Metachromatic leukodystrophy (MLD; Mendelian of Inheritance in Man \#250100) is an autosomal recessive inherited leukoencephalopathy characterized by demyelination of the central and peripheral nervous systems (1). These pathological changes are caused by failure of sulfatides and other glycolipid-containing sulfuric acids to be desulfurized and their subsequent deposition in the lysosomes of systemic tissues. Desulfurization requires the combined efforts of the enzyme arylsulfatase A (ASA; Enzyme Commission no. 3.1.6.8), encoded by the ARSA gene, a lysosomal hydrolase, and sphingolipid activator protein B, encoded by the PSAP gene. A deficiency in any of these components results in abnormal metabolism of sulfatides, which triggers progressive degenerative metabolic encephalopathy $(2,3)$. The worldwide prevalence of MLD is estimated to be between 1 in 40,000 and 1 in 170,000 (4).

MLD is divided into three clinical subtypes according to the age at onset of the disease: Late infantile ( $<4$ years), juvenile (4-14 years) and adult ( $>14$ years) (5). Clinical manifestations of MLD are varied and lack specificity (6). Three types of MLD are differentiated by the presence or absence of neurological symptoms and the pattern of disease progression. The severity of MLD appears to be negatively correlated with the age at onset (7). Infantile MLD is the most common form, accounting for $\sim 60 \%$ of all diagnosed cases. The first clinical symptoms of infantile MLD are mainly the deterioration of the motor system, including spastic tetraparesis, frequent falls and walking on toes. As the disease progresses, a patient may develop flaccid paralysis and lose the ability to stand, exhibit speech deficits and a decrease in psychological functions, develop optic atrophy, suffer generalized or partial seizures and develop peripheral neuropathy, causing death within 2-4 years from initial diagnosis (8-10). The symptoms of juvenile MLD include poor intellectual capabilities, emotional problems, language disorders and a gradual regression in motor function. Patients with juvenile MLD die within 10-15 years after the first symptoms appear, with only few cases reaching their 20 th birthday $(11,12)$. The adult form of the disease presents 
with neuromuscular or behavioural problems and progresses slowly (12).

A genotype-phenotype correlation has been reported in MLD (13-15). A deficiency in ASA is caused by mutations in the ARSA gene. The ARSA gene (GenBank accession no. NG_009260) is located on chromosome 22q13.33. This small gene $(\sim 3.5 \mathrm{~kb})$ has eight exons and encodes 509 amino acid precursors (GenBank accession nos. NM_000487.5 and NP_000478.3). Studies have indicated that in all populations, the ARSA gene has three alleles (15-18). The first allele is named ARSA-MLD (a pair of pathogenic alleles). Polten et al (15) reported that the ARSA-MLD gene has two different alleles. Mutations in one allele, designated allele I (or O), result in non-functional activity of ASA. Mutations in the other allele, designated allele A (or R), maintain the residual activity of ASA. Patients who are homozygous for allele I or allele A mainly present with the late infantile form or the adult form of MLD, respectively. Patients who are heterozygous for the two alleles (I/A) usually present with the juvenile form (6). The second allele is a pair of ASA pseudodeficiency (PD) alleles (ARSA-PD). Whether the mutation is homozygous (ARSA-PD/ARSA-PD) or heterozygous (ARSA-PD/ARSA-MLD) (17), the level of ASA may be $5-15 \%$ of the reference value in certain patients with the mutant ARSA-PD gene, while the structure of ASA is moderately altered (19). In patients with a low level of ASA, residual enzyme activity is sufficient for normal physiological functions, which is known as PD. In European and American populations, the most common ARSA-PD allele has two variants (16). One is c.1049A $>\mathrm{G}(\mathrm{N} 350 \mathrm{~S})$, which prevents the ASA protein from entering lysosomes. The other is c.*96A $>\mathrm{G}$, a mutation in the $3^{\prime}$ untranslated region (poly A tail), which causes a significant reduction in ASA protein production. However, Asian populations have only one allele (c.1049A>G) of ARSA-PD. These Asian patients account for 20-30\% of all patients with MLD (20). Furthermore, homozygosity for the mutant gene has little effect on the activity of ASA. The third allele is (ARSA-MLD; ARSA-PD) (18). It is reported that ARSA-MLD and ARSA-PD, known as the ARSA-MLD-PD allele, are located on the same chromosome. Based on the aforementioned genetic heterogeneity of MLD, it is required to identify this multiple allele in the ARSA gene to determine the genotype-phenotype correlation. To date, 254 mutations have been detected in the ARSA gene (21).

In the present study, whole-exome sequencing (WES) was performed in the family of a case of MLD to identify causative mutations and to offer a potential explanation for the genotype-phenotype correlation in MLD.

\section{Subjects and methods}

Subjects and clinical evaluation. The patient of the present study was recruited at Yuying Children's Hospital of Wenzhou Medical University (Wenzhou, China). At the first medical examination of the male patient at the age of 2 years 8 months due to a motor vehicle collision without any obvious indication of resulting neurological damage, a brain magnetic resonance imaging (MRI) scan was performed to ensure no underlying brain injury. The imaging unexpectedly revealed signs of MLD. Further enquiries regarding the proband's family history unveiled that the proband's grandparents (maternal parents) had a consanguineous marriage. Given the special family history, cognitive assessments were performed using the Gesell Development Scales test (22). It is the most common test to assess neurobehavioural development in infants ( $<3$ years of age) through five factors (gross motor function, fine motor function, speech, adaptive behaviour and interpersonal behaviour). The results of Gesell Development Scales test was determined as development quotient (DQ) (22). The patient was evaluated by determining the DQ at the age of 2 years 8 months. Pediatric subjects scoring DQ $\leq 75,75<\mathrm{DQ}<85$ and $\mathrm{DQ} \geq 85$ exhibit low, marginal and normal development, respectively (23). In addition, several complementary inspections were performed to confirm the diagnosis, including ASA activity in leukocytes, electromyography (EMG) and gas chromatography-mass spectrometry (GC-MS) (24) at the proband's first visit. GC-MS represents an unbiased and open approach that allows the detection of unexpected changes in metabolite levels.

During 6 years of follow-up, the patient was subjected to continuous physical assessments. The Wechsler Intelligence Scale-third Edition (WAIS-III) test for neurodevelopmental assessment $(25,26)$ was performed when the patient turned 4 years old. At our institution, the WAIS-III test is commonly used to evaluate the neurodevelopmental status of individuals aged $\geq 4$ years (26). The results of the WAIS-III test are expressed as an intelligence quotient (IQ), and the IQ is scored according to the UK WAIS-III manual scoring criteria (25). An IQ of 90 is the boundary between normal and low intelligence. The WAIS-III comprises 14 subtests (vocabulary, similarities, information, comprehension, block design, matrix reasoning, picture completion, picture arrangement, coding, symbol search, digit span, letter-number sequencing, arithmetics and object assembly) (27). The Ayres Sensory Integration (ASI) test (28-30) was performed at the age of 7 years 11 months (July, 2017) to assess behavioural problems. Furthermore, neuroradiological studies were performed by brain MRI scans as the patient aged. At the most recent visit, the proband and the proband's parents were subjected to genetic testing.

WES. Genomic DNA was isolated from peripheral blood leukocytes of the affected proband and the proband's parents by Kangso Medical Inspection. Whole-exome capture using the SureSelect Human All Exon kit (Agilent Technologies, Inc.) and high-throughput sequencing were performed in-house as previously described $(7,8)$. The reads were aligned for single-nucleotide variant (SNV) calling and subsequent analysis for prioritization of candidate genes (9). ANNOVAR (version 20180118) (31) was used to annotate the detected variations. ANNOVAR can utilize annotation databases conforming to Generic Feature Format version 3 (GFF3). The gene mutations we verified based on the low frequency of the detected variants provided in relevant gene databases [mainly the 1000 Genomes Project (https:/www.ncbi.nlm.nih. gov/variation/tools/1000genomes/) and dbSNP (https://www. ncbi.nlm.nih.gov/SNP/)]. First, DNA samples were compared with the hy 19 reference (one version of normal human genome from NCBI database). If the detected mutations did not match the template file, a database was consulted (mainly the 1000 Genomes Project). Subsequently, according to the frequency 


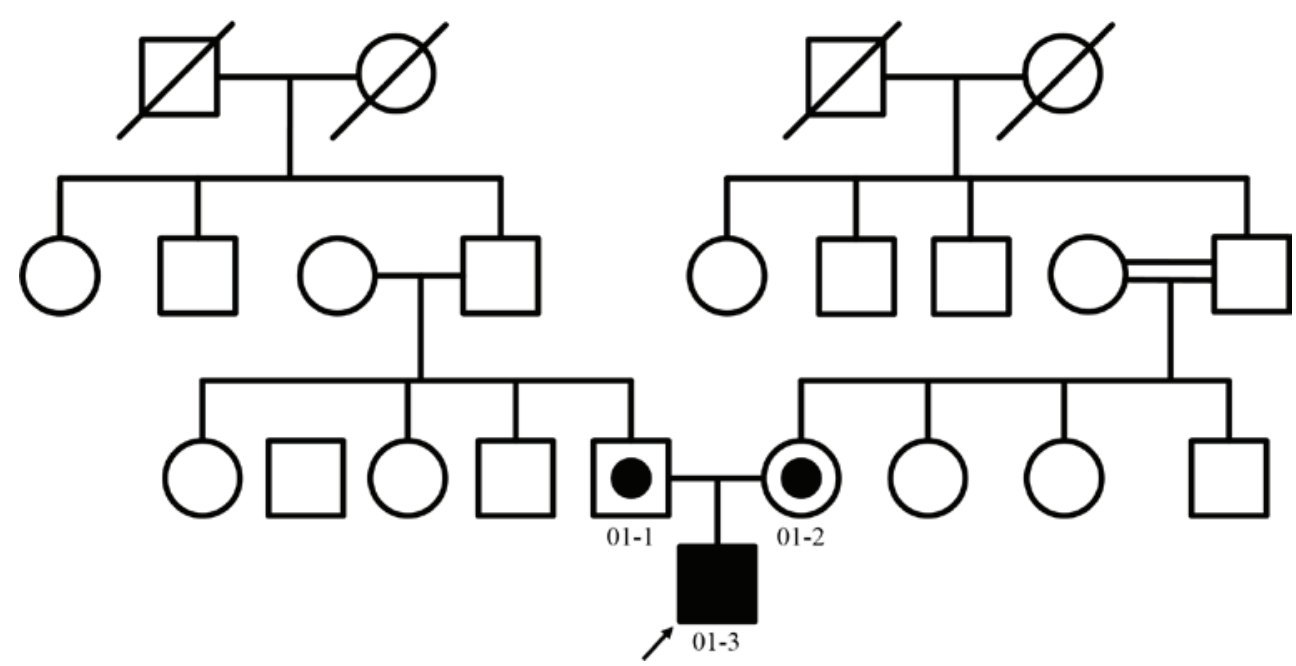

Figure 1. Pedigree of the case of MLD. Squares represent males and circles represent females. The proband is indicated by an arrow. The shaded symbol indicates the affected individual. A dot within the symbol represents carrier status for ARSA mutations. A double line between a square and a circle represents consanguinity. 01-3 is the proband and affected individual, whereas the proband's mother (01-1) and father 01-2) were carriers for the c.1297C $>\mathrm{G}$ and c.1345G>A mutations, respectively. MLD, metachromatic leukodystrophy disorder.

of base mutations given in the database, it was determined whether the mutation site is pathogenic. Furthermore, several studies suggest that in numerous cases, variations in the chromosome copy number $(\mathrm{CNV})$, rearrangement and structure may be connected with disease (32). Therefore, the CNV was also determined by using CODEX (33) and XHMM software (34). CODEX and XHMM present a novel normalization and CNV calling method. CODEX was used to remove biases and artifacts in WES data, and produce accurate CNV calls. The XHMM software was designed to recover information on CNVs from targeted exome sequence data.

In silico analysis. Deleterious missense SNVs were predicted by the following web-based tools: i) Sorting Intolerant From Tolerant (SIFT; sift.bii.a-star.edu.sg/), where an SNP with a SIFT score $<0.05$ predicts a negative effect on the encoded amino acid, ii) polyphen-2 (genetics.bwh.harvard.edu/pph2/), where an SNP with a score between 0.85 and 1.0 predicts a damaging effect on the encoded amino acid, while an SNP with a score between 0.0 and 0.15 is predicted to be benign and an SNP with a score between 0.15 and 1.0 predicts a possibly damaging function, and iii) Mutation Taster (www. mutationtaster.org/), where a score close to 1 indicates a high 'security' of the prediction of the given variant to be disease-causing.

In addition, multiple phylogenetic tree analyses of ARSA homologs were performed using MEGA7 software (35).

\section{Results}

Clinical manifestation. A Chinese pedigree from the city of Wenzhou with an index patient (MLD01) was recruited for the study (Fig. 1). The diagnosis of MLD was made pre-symptomatically at the age of 2 years 8 months based on the typical presentation of brain MRI. At first, the patient was asymptomatic with generally normal development. Later, the proband exhibited mild behavioural problems and was diagnosed with attention deficit hyperactivity disorder (ADHD) at the age of 7 years 11 months based on the ASI examination.
He had a normal gait with no signs of paraparesis, spasticity or neuropathy, but was socially immature based on his interactions with the surroundings and individuals, as well as the ASI test. He was in a relatively stable pre-onset stage at the time of writing. The result of the EMG was normal. To date, the proband has developed no other neurological symptoms and lives an ordinary life, except for suffering from ADHD and struggling to keep up with his classmates in a regular school.

ARSA activity determination. No biochemical abnormalities were detected in the proband. ARSA activity in leukocytes of the proband was $150 \mathrm{nmol} / 17 \mathrm{~h} / \mathrm{mg}$ protein at the first visit (at the age of 3 years in 2012) and $142 \mathrm{nmol} / 17 \mathrm{~h} / \mathrm{mg}$ protein at the most recent visit (at the age of 8 years in 2017). Notably, the activity of ASA in 2017 was at the lower end of the normal range (normal range: $134.1-325.1 \mathrm{nmol} / 17 \mathrm{~h} / \mathrm{mg}$ protein).

Neurodevelopmental assessment. At the first visit at the age of 2 years 8 months in March 2012, testing on the Gesell Development Scale revealed a borderline DQ level (75.1). At 4 years of age (in 2013), WAIS-III testing (36) suggested that the patient had mild mental retardation (IQ, 81). Neurocognitive evaluation in 2014 indicated a slight abnormality in full-scale IQ with slow processing, low average working memory and below-average executive skills. On the last follow-up (in 2017), the IQ had reached an almost stable level at 80. In addition, ASI testing suggested that the patient presented with inattention and bad temper, and the patient was subsequently diagnosed with ADHD. Given the mild symptoms, it was suggested that the patient could focus on home education. The parents could have greater patience and provide sufficient attention to the child. In addition, attention exercises, including handwriting, were encouraged.

Neuroradiological studies. The images of the first MRI scan (Fig. 2Aa-c) in March 2012 exhibited a lightly enlarged lateral ventricle and sporadic tigroid pattern of white matter in the centrum semiovale and basal ganglia. These results 

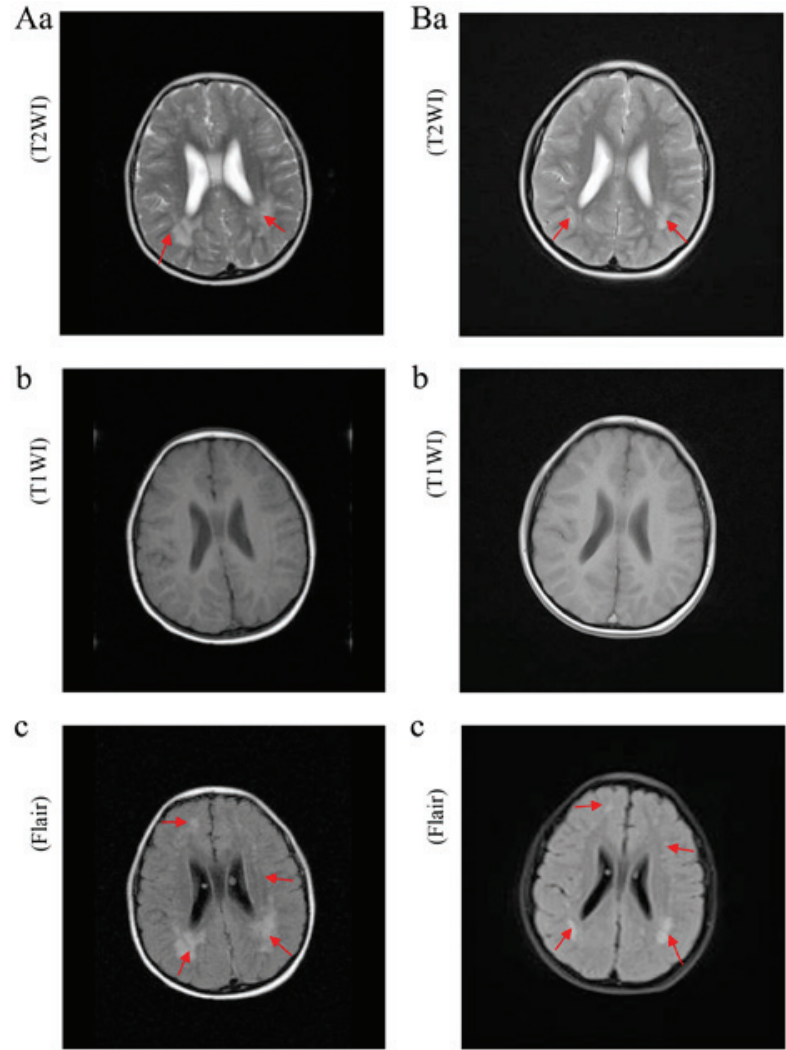

Figure 2. Brain magnetic resonance images. (A) Axial (a) T2WI, (b) T1W and (c) FLAIR images of the proband at the age of 2 years 8 months, indicating a tigroid pattern of white matter at the level of the centrum semiovale and basal ganglia hyperintensity (red arrows). (B) Axial (a) T2WI, (b) T1WI and (c) FLAIR images of the proband at the age of 7 years 11 months had almost the same characterization. The red arrows represent abnormal signal in the brain (a tigroid pattern of white matter at the level of the centrum semiovale and basal ganglia hyperintensity) T1WI, T1-weighted image; FLAIR, fluid-attenuated inversion recovery.

are characteristic of MLD. On the last MRI scan (Fig. 2Ba-c) in 2017, white-matter hyperintensities were effectively unchanged. Over the 6-year follow-up period, brain MRI scans revealed no evidence of progressive demyelination, indicating that the patient is in a stable condition.

Mutation detection. The mutation frequency may be expressed as that in Asian populations (ASN) or in East Asians (EAS). The mutation frequency of ' $c .1297 \mathrm{C}>\mathrm{G}$ ' in the ARSA gene of the proband was $\mathrm{ASN}=0.0035$ and $\mathrm{EAS}=0.0020$, respectively, and the mutation frequency of ' $c .1345 \mathrm{G}>\mathrm{A}$ ' in the ARSA gene of the proband was identical (ASN=0.0035 and $\mathrm{EAS}=0.0020$ ). Therefore, after variant filtering, the study focused on the compound heterozygous variants (c.1297C $>\mathrm{G})+(\mathrm{c} .1345 \mathrm{G}>\mathrm{A})$ in exon 8 in the ARSA gene of the patient, which result in p.Leu433Val and p.Gly449Arg amino acid substitutions, respectively. Sanger sequencing was performed to confirm the above results and to investigate the possible familial segregation of the mutations. Compound heterozygous mutations detected in the proband's parents indicated that each of the proband's parents was a heterozygous carrier of either of the two mutations (Fig. 3), who coincidentally passed the mutation on to the proband individually. Furthermore, no mutations with an autosomal-dominant pattern of inheritance were identified.

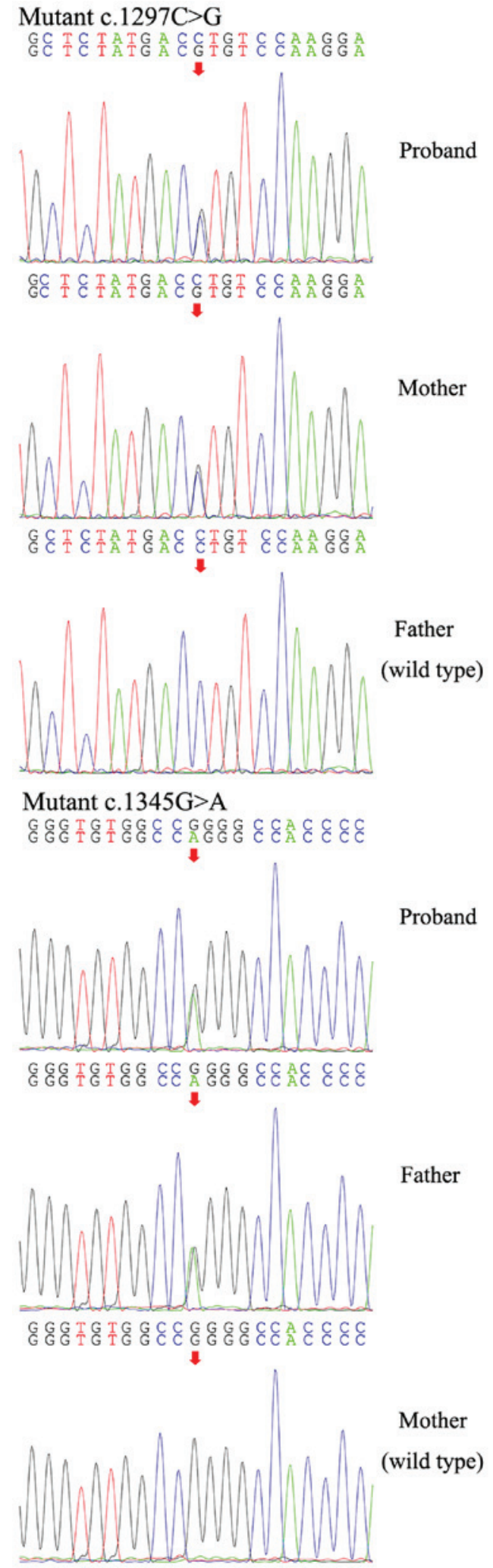

Figure 3. DNA sequencing analysis of mutations in the ARSA gene. Mutants c. $1297 \mathrm{C}>\mathrm{G}$ and c. $1345 \mathrm{G}>\mathrm{A}$ in exon 8 of ARSA compared with wild-type and heterozygous sequences are indicated with a red arrow.

In addition, no suspicious CNVs were identified in the WES data through the aforementioned computational methods.

In silico analysis. To further characterize the missense mutations identified in the family, different in silico tools 
Table I. Pathogenicity predictions for p.Leu433Val and p.Gly449Arg mutations in ARSA by online in silico prediction tools.

\begin{tabular}{llll}
\hline Amino acid substitution & SIFT $^{\mathrm{a}}$ & PolyPhen-2 $^{\mathrm{b}}$ & Mutation Taster $^{\mathrm{c}}$ \\
\hline p.Leu433Val & Tolerated & Probably damaging & Disease-causing \\
p.Gly449Arg & Tolerated & Benign & - \\
\hline
\end{tabular}

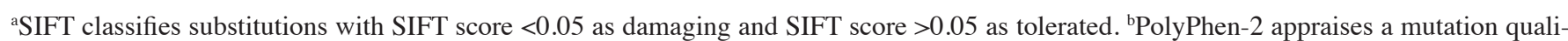
tatively, as benign, possibly damaging or probably damaging based on pairs of false-positive rate thresholds. ${ }^{c}$ Mutation Taster score using the following rule: If the prediction is 'disease-causing' or 'disease-causing-automatic', score=P-value; if the prediction is 'polymorphism' or 'polymorphism-automatic', score=1-P-value. The resulting score ranges from 0 to 1 and a larger score indicates a greater likelihood of the mutation being deleterious. SIFT, Sorting Intolerant From Tolerant.

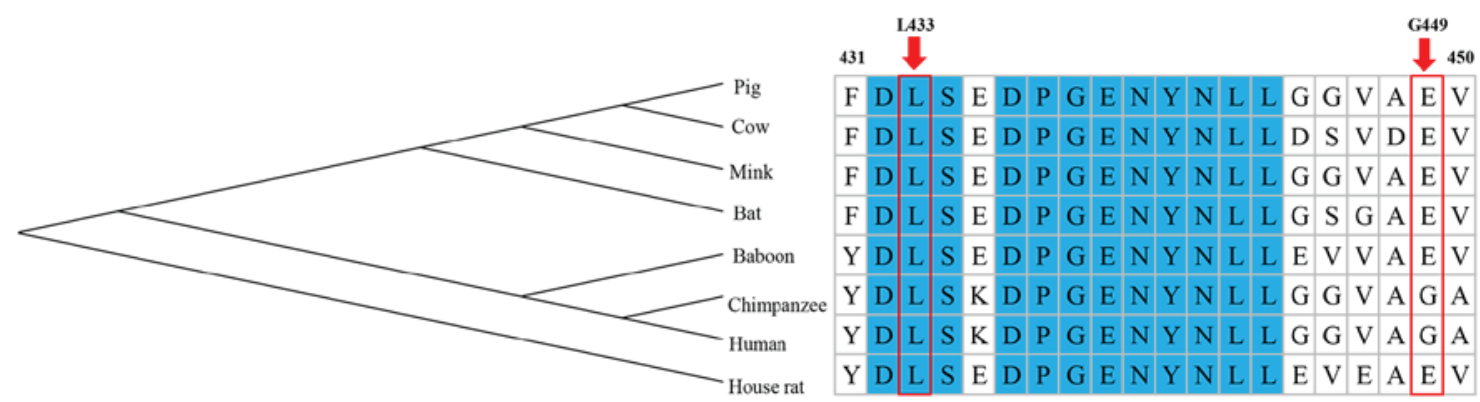

Figure 4. MSA. Phylogenetic tree and MSA displaying the sequence alignment of two specific amino acids, as indicated individually by red arrows, and conservation of each in other ARSA orthologues (across different species). The first red arrow indicates the conservation of amino acid L in all species at position 433 and the second represents amino acid $\mathrm{G}$ at position 449. MSA, multiple sequence alignment; L, leucine; G, glycine.

were used to assess the influences that the novel c.1297C $>\mathrm{G}$ and c. $1345 \mathrm{G}>\mathrm{A}$ missense mutations have on ARSA protein function. Pathogenicity predictions by the Mutation Taster programme suggested that the $c .1297 \mathrm{C}>\mathrm{G}$ mutation is deleterious (Table I). Pathogenicity predictions by the SIFT and PolyPhen-2 programme suggested that the c.1297C>G mutation is tolerated and probably damaging, respectively. The accuracy of Mutation Taster is reportedly the highest of the three utilized tools (37).

Furthermore, it is thought that highly conserved amino acid sequences have a functional value and are significant for protein structure, which indicates that they have an important role in determining the conformation of different domains of a protein (38). Multiple sequence alignment of ARSA orthologues revealed that the amino acid L433 is highly conserved across mammalian species, from human to rat, and the amino acid G449 is less conserved. The evolutionary conservation of the amino acid L433 indicates its significance in the structure of the ASA protein (Fig. 4).

\section{Discussion}

The present study reported on a Chinese pediatric patient diagnosed with MLD manifesting as ADHD as the major clinical symptom. Of note, two missense mutations were identified in the ARSA gene of the patient. The compound heterozygous variants in the proband [(c.1297C $>\mathrm{G})+(\mathrm{c} .1345 \mathrm{G}>\mathrm{A})$ ] were inherited from the proband's mother and father, respectively. Of note, no de novo variants were detected in the proband. According to software prediction and comparison of homologous proteins, C.1297 was indicated to be the major mutation contributing to the onset of the disease. By contrast, the patient's mother, who possesses the same mutation, is normal. From the phenotype, the proband had a tigroid pattern on every brain MRI and was diagnosed with mild behavioural problems with MLD, whereas the proband's parents had no symptoms. Notably, the subtype of adult MLD ( $>14$ years) is characterized by neuromuscular or behavioural problems. However, the reported case showed behavioural problems at the age of 7 years. Therefore, it is likely that this presentation was due to the compound heterozygous variants $[(\mathrm{c} .1297 \mathrm{C}>\mathrm{G})+(\mathrm{c} .1345 \mathrm{G}>\mathrm{A})]$.

Biochemical analysis indicated that the activity of ASA was normal in the proband, which indicates that the mutations in the two genes did not affect the enzyme level. Although test for ASA activity in 2017 was at the lower end of the normal range, ASA activity remained normal in patient's body. That is, there is no qualitative change in ASA. Combined with the results of a previous study, it may be hypothesized that c. $1297 \mathrm{C}>\mathrm{G}$ and $\mathrm{c} .1345 \mathrm{G}>\mathrm{A}$ probably belong to mutations in the ARSA-PD allele. It is reported that the compound heterozygous mutation in ARSA-PD/ARSA-MLD is not associated with progressive neurological disease (17).

Neurologically, all brain MRI scans for the proband display pathological changes of demyelination, which is characteristic of MLD. Furthermore, the patient's MRI images exhibit little progression during the 6-year follow-up period. It may be hypothesized that this may be associated with the normal enzyme levels in the proband.

Concerning the phenotype, the patient's condition cannot be regarded as severe when compared to other cases of MLD. 
Given that the disease is usually classified according to the age of onset, it is difficult to classify this case into a proper subtype of MLD. The patient presented with ADHD-like symptoms but had no neurological signs at the age of 7 years 11 months. The MRI images exhibited demyelination of the cerebrum at the age of 2 years 8 months. If this case was to be classified according to the age of onset, the patient should be diagnosed with juvenile MLD, according to the clinical symptoms, the patient, who displayed behavioural problems, should be diagnosed with the adult form. Therefore, subjects with suspicion of MLD should be comprehensively evaluated. The clinical manifestation of MLD is highly variable. If clinicians diagnosed MLD only based on the general indicators of the three subtypes, numerous patients would be misdiagnosed or the diagnosis would be missed. Subjects with suspected MLD, particularly in a consanguineous family, may be diagnosed through genetic testing. In additionally, the phenotype is the result of interactions between genes and the environment. A tigroid pattern of white matter at the level of the centrum semiovale and basal ganglia hyperintensity indicated in MRI highly suggests a diagnosis of MLD, especially when considering the consanguinity relationship between the child's grandparents. The clinical presentation of the proband of the present study is atypical, which may be associated with his living environment. Of note, he possessed a large number of good educational resources and his parents provided a nurturing environment and sufficient attention.

However, the present study only included one family. It may be worthwhile to perform a cohort study to further examine genotype-phenotype associations in MLD. In addition, experimental animal studies with the same mutations are required to investigate the biochemical characterization of MLD (39). By enlarging the number of samples for mutation analysis, it is possible to screen those subjects at risk or with symptoms to diagnose early. The authors of the current study may be able to summarise some common features by increasing the size of the cohort in future studies. As physician can recognize people at high risk of MLD, only individual gene test need to be performed rather than WES. This is helpful for detecting ARSA gene mutations quickly and reliably, and reducing the cost of genetic testing.

In conclusion, the compound heterozygous variants (c.1297C > G $)+($ c.1345G $>A)$ were described in a Chinese pediatric patient with MLD. The patient, having normal activity of ASA, presented with ADHD-like symptoms, no neurological symptoms and obvious signs of MLD on MRI.

\section{Acknowledgements}

Not applicable.

\section{Funding}

No was funding received.

\section{Availability of data and materials}

Not applicable.

\section{Authors' contributions}

$\mathrm{XC}$ and YYW contributed the central idea and analyzed most of the data. YYW wrote the initial draft of the paper. CL, SMW, QFX, QH, SC and YWL contributed to refining the ideas, carrying out additional analyses and finalizing this paper.

\section{Ethical approval and consent to participate}

Informed consent was obtained from the proband's parents. The present study was approved by the ethics committee of Wenzhou Medical University (Wenzhou, China).

\section{Patient consent for publication}

The patient's parents were contacted by telephone to obtain verbal informed consent.

\section{Competing interests}

The authors declare that they have no competing interests.

\section{References}

1. Coulter-Mackie MB , Gagnier L , Beis MJ, Applegarth DA, Cole DEC, Gordon K and Ludman MD: Metachromatic leucodystrophy in three families from Nova Scotia, Canada: a recurring mutation in the arylsulphatase A gene. Journal of Medical Genetics 34: 493-498, 1997.

2. Aggarwal S, Yurlova L and Simons M: Central nervous system myelin: Structure, synthesis and assembly. Trends Cell Biol 21: 585-593, 2011.

3. Bosio A, Binczek E and Stoffel W: Functional breakdown of the lipid bilayer of the myelin membrane in central and peripheral nervous system by disrupted galactocerebrosides synthesis. Proc Natl Acad Sci USA 93: 13280-13285, 1996.

4. Brimley CJ, Lopez J, van Haren K, Wilkes J, Sheng X, Nelson C, Korgenski EK, Srivastava R and Bonkowsky JL: National variation in costs and mortality for leukodystrophy patients in US children's hospitals. Pediatr Neurol 49: 156-162,e1, 2013.

5. Shahzad MA, Khaliq S, Amar A and Mahmood S: Metachromatic leukodystrophy (MLD): A pakistani family with novel ARSA gene mutation. J Mol Neurosci 63: 84-90, 2017.

6. Gieselmann V, Polten A, Kreysing J and von Figura K: Molecular genetics of metachromatic leukodystrophy. J Inherit Metab Dis 13: 222-227, 1994

7. Simell O: The metabolic and molecular bases of inherited disease. JAMA 286: 2329, 2001

8. Lugowska A, Mierzewska H, Bekiesińska-Figatowska M, Szczepanik E, Goszczańska-Ciuchta A and BednarskaMakaruk M: A homozygote for the c. $459+1 \mathrm{G}>\mathrm{A}$ mutation in the ARSA gene presents with cerebellar ataxia as the only first clinical sign of metachromatic leukodystrophy. J Neurol Sci 338: 214-217, 2014.

9. Barboura I, Ferchichi S, Dandana A, Jaidane Z, Ben Khelifa S, Chahed H, Ben Mansour R, Chebel S, Maire I and Miled A: Metachromatic leucodystrophy. Clinical, biological, and therapeutic aspects. Ann Biol Clin (Paris) 68: 385-391, 2010 (In French).

10. Kehrer C, Groeschel S, Kustermann-Kuhn B, Bürger F, Köhler W, Kohlschütter A, Bley A, Steinfeld R, Gieselmann V and Krägeloh-Mann I; German LEUKONET: Language and cognition in children with metachromatic leukodystrophy: Onset and natural course in a nationwide cohort. Orphanet J Rare Dis 9: 18, 2014.

11. Barrell C: Juvenile metachromatic leukodystrophy: Understanding the disease and implications for nursing care. J Pediatr Oncol Nurs 24: 64-69, 2007.

12. Patil SA and Maegawa GH: Developing therapeutic approaches for metachromatic leukodystrophy. Drug Des Devel Ther 7: 729-745, 2013. 
13. Gieselmann V, Fluharty AL, Tønnesen T and Von Figura K: Mutations in the arylsulfatase A pseudodeficiency allele causing metachromatic leukodystrophy. Am J Hum Genet 49: 407-413, 1991.

14. Regis S, Corsolini F, Stroppiano M, Cusano R and Filocamo M: Contribution of arylsulfatase A mutations located on the same allele to enzyme activity reduction and metachromatic leukodystrophy severity. Hum Genet 110: 351-355, 2002.

15. Polten A, Fluharty AL, Fluharty CB, Kappler J, von Figura K and Gieselmann V: Molecular basis of different forms of metachromatic leukodystrophy. N Engl J Med 324: 18-22, 1991.

16. Tinsa F, Caillaud C, Vanier MT, Bousnina D, Boussetta K and Bousnina S: An unusual homozygous arylsulfatase: A pseudodeficiency in a metachromatic leukodystrophy tunisian patient. J Child Neurol 25: 82-86, 2010.

17. Penzien JM, Kappler J, Herschkowitz N, Schuknecht B, Leinekugel P, Propping P, Tønnesen T, Lou H, Moser H, Zierz S, et al: Compound heterozygosity for metachromatic leukodystrophy and arylsulfatase A pseudodeficiency alleles is not associated with progressive neurological disease. Am J Hum Genet 52: 557-564, 1993.

18. Fluharty AL: Arylsulfatase A Deficiency-GeneReviews®-NCBI Bookshelf. University of Washington Seattle, 2014.

19. Aubourg P, Sevin C and Cartier N: Mouse Models of Metachromatic Leukodystrophy and Adrenoleukodystrophy. In: De Deyn P, Van Dam D (eds) Animal Models of Dementia. Neuromethods, Humana Press 48: 493-513, 2011.

20. Shi H: Metachromatic Leukodystrophy. Chinese Journal of Practical Pediatrics 507-510, 2009 (In Chinese).

21. The Human Gene Mutation Database at the Institute of Medical Genetics in Cardiff: http://www.hgmd.cf.ac.uk/ac/gene. php?gene=ARSA. Accessed, April 23, 2019.

22. Fishler K, Graliker BV and Koch R: The predictability of intelligence with gesell developmental scales in mentally retarded infants and young children. Am J Ment Defic 69: 515-525, 1965.

23. Huang XN, Zhang Y, Feng WW, Wang HS, Cao B, Zhang B Yang YF, Wang HM, Zheng Y, Jin XM, et al: Reliability and validity of warning signs checklist for screening psychological, behavioral and developmental problems of children. Zhonghua Er Ke Za Zhi 55: 445-450, 2017 (In Chinese)

24. Roessner U, Wagner C, Kopka J, Trethewey RN and Willmitzer L: Technical advance: Simultaneous analysis of metabolites in potato tuber by gas chromatography-mass spectrometry. Plant J 23: $131-142,2010$

25. Dumont R and Willis JO: Wechsler Adult Intelligence Scale-3rd edition. John Wiley, Sons, Inc, 2008

26. Wechsler D: Wechsler Adult Intelligence Scale-3rd edition, 1997.

27. Cockcroft K, Alloway T, Copello E and Milligan R: A cross-cultural comparison between South African and British students on the Wechsler Adult Intelligence Scales Third Edition (WAIS-III). Front Psychol 6: 297, 2015.
28. Mailloux Z, Mulligan S, Roley SS, Blanche E, Cermak S, Coleman GG, Bodison S and Lane CJ: Verification and clarification of patterns of sensory integrative dysfunction. Am J Occup Ther 65: 143-151, 2011.

29. Mulligan S: Patterns of sensory integration dysfunction: A confirmatory factor analysis. Am J Occupational Therapy 52: 819-828, 1998

30. Parham LD, Roley SS, May-Benson TA, Koomar J, Brett-Green B, Burke JP, Cohn ES, Mailloux Z, Miller LJ and Schaaf RC. Development of a Fidelity Measure for Research on the Effectiveness of the Ayres Sensory Integration(R) Intervention[J]. American Journal of Occupational Therapy 65: 133-142, 2011.

31. Wang K, Li M and Hakonarson H: Hakonarson, ANNOVAR: Functional annotation of genetic variants from high-throughput sequencing data. Nucleic Acids Res 38: e164, 2010.

32. Carter NP: Methods and strategies for analyzing copy number variation using DNA microarrays. Nat Genet 39 (7 Suppl): S16-S21, 2007.

33. Jiang Y, Oldridge DA, Diskin SJ and Zhang NR: CODEX: A normalization and copy number variation detection method for whole exome sequencing. Nucleic Acids Res 43: e39, 2015.

34. Fromer M and Purcell SM: Purcell, using XHMM software to detect copy number variation in whole-exome sequencing data. Curr Protoc Hum Genet 81: 7.23.1-21, 2014.

35. Tamura K, Peterson D, Peterson N, Stecher G, Nei M and Kumar S: MEGA5: Molecular evolutionary genetics analysis using maximum likelihood, evolutionary distance, and maximum parsimony methods. Mol Biol Evol 28: 2731-2739, 2011.

36. Iverson GL: Interpreting change on the WAIS-III/WMS-III in clinical samples. Arch Clin Neuropsychol 16: 183-191, 2001.

37. Schwarz JM, Cooper DN, Schuelke M and Seelow D: MutationTaster2: Mutation prediction for the deep-sequencing age. Nat Methods 11: 361-362, 2014

38. Shahzad MA, Khaliq S, Amar A and Mahmood S: Metachromatic leukodystrophy (MLD): A pakistani family with novel ARSA gene mutation. J Mol Neurosci 63: 84-90, 2017.

39. Özkan A and Özkara HA: Metachromatic leukodystrophy: Biochemical characterization of two (p.307Glu->Lys, p.318Trp- $>$ Cys) arylsulfatase A mutations. Intractable Rare Dis Res 5: 280-283, 2016.

This work is licensed under a Creative Commons Attribution-NonCommercial-NoDerivatives 4.0 International (CC BY-NC-ND 4.0) License. 